\section{Rechtssicherer Schutz für ausgelagerte Daten}

Ärzte, die ihre Patientendaten einem externen Dienstleister anvertrauen, etwa zur Archivierung, haften persönlich dafür, dass die Daten nicht in falsche Hände geraten - bis hin zur Beschlagnahme durch den Staat. Der E-Health-Anbieter CompuGroup Medical hat jetzt ein patentiertes Verfahren entwickelt, das einen technischen Beschlagnahmeschutz gewährleistet. Die Daten werden dabei schon vor dem Versand an andere Stellen verschlüsselt. Sie seien damit für alle potenziellen Interessenten - außer für Ärzte und ihre Patienten - unleserlich und damit unbrauchbar, teilt das Unternehmen mit.

\section{Per Bausteinsystem zur} eigenen Praxis-Homepage

Das Internet wird auch für Ärzte als Kommunikationsinstrument immer wichtiger. Immerhin knapp 70\% der Deutschen sind laut der ARD/ZDF-Onlinestudie 2010 zumindest gelegentlich im Internet unterwegs. Da ist eine eigene Praxis-Homepage sinnvoll. Einen einfachen Weg zu dieser Homepage bietet die 1 \& 1 Internet AG (www.1 und1. info/gruender). Über ein Baukastensystem lässt sich die Praxishomepage schnell und ohne Programmierkenntnisse aufbauen. Dabei ist die Lösung über den eigenen Web-Browser zugänglich und kann im ersten Jahr kostenlos getestet werden.

\section{Lokale Applikationen auch} virtuell nutzen

In virtuellen Umgebungen gleichzeitig auf lokale und virtuelle Anwendungen zuzugreifen, kann mitunter Schwierigkeiten bereiten. Helfen soll hier der RES Virtual Desktop Extender (VDX), den das IT-Unternehmen RES Software nun auch als eigenständiges Produkt veröffentlicht hat. Der VDX sorgt laut Unternehmensangaben dafür, dass Anwender auch lokale Anwendungen unterbrechungs- und verzögerungsfrei in virtuelle Desktop-Sessions einbinden und so auf lokale Daten zugreifen können. Das Programm sei mit marktführenden Desktop-Virtualisierungslösungen wie etwa Citrix XenApp, Citrix XenDesktop, VMwareView und Microsoft Remote Desktop Services kompatibel.

\title{
Wenn die Praxis-EDV mit auf Hausbesuch geht
}

\author{
Papierdokumente mitschleppen und hinterher in der Praxis alles ab- \\ tippen? Das ist heutzutage nicht mehr nötig. Denn die Praxis-EDV \\ geht via Notebook, USB-Stick, iPad oder iPhone einfach mit auf Haus- \\ besuch. Welche Technik ist dabei die richtige für die Praxis?
}

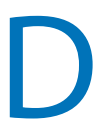
ie Zeiten, in denen Ärzte auf Heim- oder Hausbesuch ihre Dokumentation zunächst händisch vornehmen mussten, um die Daten dann in der Praxis entweder selbst oder durchs Personal in die EDV eingeben zu lassen, sind vorbei. Die Praxisverwaltungssysteme (PVS) sind längst so weit, dass sie als mobile Lösung den Arzt nahezu überall hin begleiten können.

Die Fragen, die sich Ärzte heute aber stellen, sind: Soll ich die Daten online oder offline nutzen? Und für welche HardwareVariante soll ich mich entscheiden: Notebook, iPad, iPhone oder doch eine Lösung via USB-Stick? Ein Blick hinter die Technik hilft bei der Qual der Wahl.

Zunächst einmal gibt es die eher klassische Lösung, die auf Notebooks hin ausgerichtet ist. Hier werden einzelne Patientendaten oder auch der komplette aktuelle Datenbestand der Praxis auf das Gerät übertragen. Wobei in der Regel alle PVS-Funktionen wie Arzneimittel-Checks, Abrechnungshilfen und Textbausteine genutzt werden können. „Es ist keine Online-Anbindung nötig“, erklärt Lars Wichmann, Geschäftsführer der Frey $\mathrm{ADV} \mathrm{GmbH}$, die eine solche Lösung für Quincy win anbietet. „Sobald der Arzt mit seinem Notebook zurück in die Praxis kommt, schließt er das Notebook wieder an die Praxis-EDV-Anlage und synchronisiert die Daten mit dem Praxisserver."

Ein schweres Notebook in die Besuchstasche packen, das muss nicht mehr sein, denn die PraxisEDV läuft auch auf dem iPad.

\section{Synchron Arbeiten in Praxis und auf Besuch}

Bei Quincy win und auch bei TurboMed oder etwa S3 stehen dabei alle Praxisdaten zur Verfügung. Das MedistarHausbesuchsmodul hingegen arbeitet mit einer Besuchsliste.

Hier überträgt der Arzt lediglich die Daten seiner Hausbe-

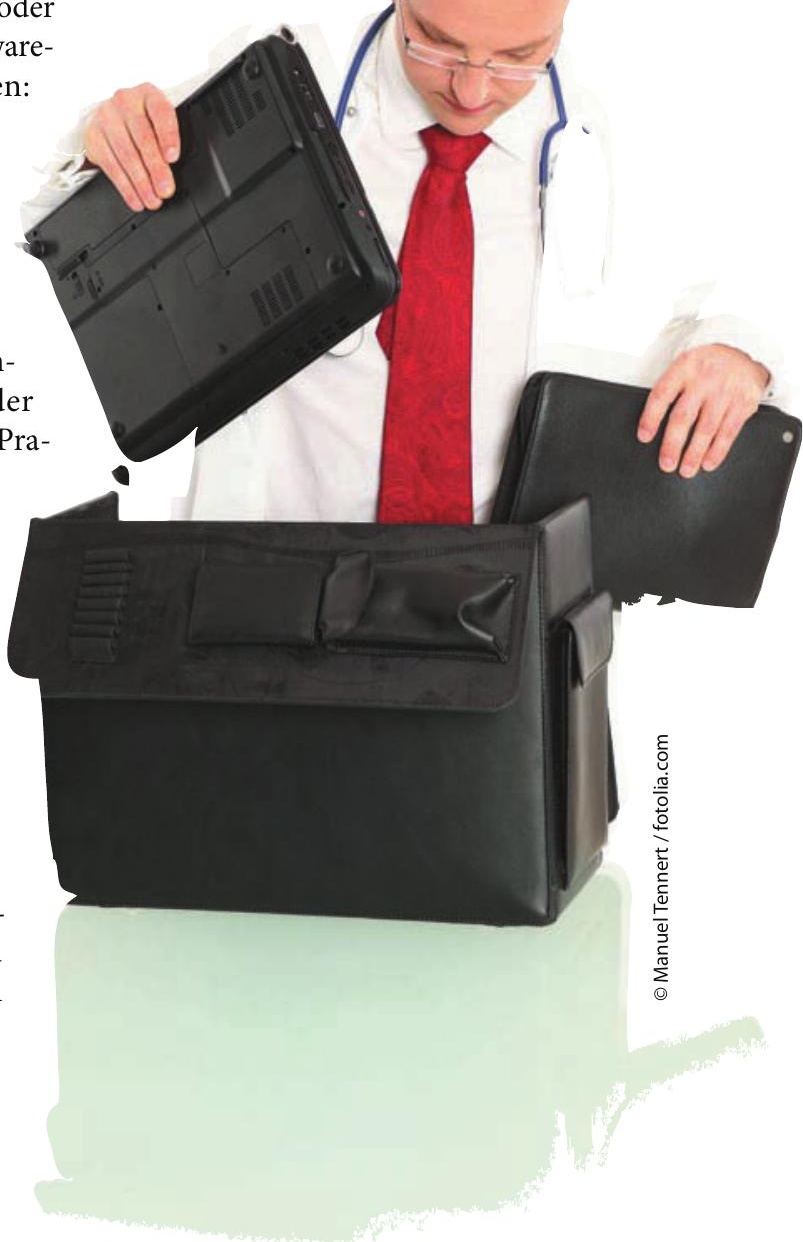

